\title{
VALORACIÓN DEL GRADO DE ADAPTACIÓN DE LOS PLANES SUBREGIONALES ANDALUCES A LA DIRECTIVA MARCO DE AGUAS
}

\author{
Mercedes Rosa España Villanueva \\ Laboratorio de Planificación Ambiental (LABPLAM) \\ Departamento de Urbanística y Ordenación del Territorio-Universidad de Granada \\ merespavil@ugr.es
}

\section{RESUMEN}

Entre las novedades que introdujo la Directiva Marco del Agua (DMA) está el reconocimiento legislativo de la necesidad de integrar las políticas del agua y del territorio. La incorporación del enfoque hidrológico en los planes territoriales y viceversa se ha identificado como fórmula para avanzar hacia el objetivo de la integración. En Europa, esta idea implica que los planes territoriales asuman los principios, objetivos y medidas de la propia Directiva. Este artículo pretende realizar un diagnóstico acerca del grado de adaptación de cinco planes territoriales subregionales andaluces a la Directiva, en base a la propuesta de una serie de criterios evaluadores.

Palabras clave: Directiva Marco del Agua, criterios, planificación territorial subregional.

\begin{abstract}
The recognition of the need to integrate water and territorial policies was one of the innovations introduced by the Water Framework Directive (WFD). A possible way to advance toward integration policies is the incorporation of hydrological approach into the spatial planning and vice versa. This idea, in Europe, implies that spatial plans should assume the principles, objectives and measures of the Directive. The present paper aims at achieving an assessment of the adaptation degree to the Directive by five specific Andalusian Sub-regional Plans. To this end, the paper proposes ten evaluation criteria.
\end{abstract}

Keywords: Water Framework Directive criteria, sub-regional plans.

Fecha de recepción: julio 2014.

Fecha de aceptación: febrero 2015. 


\section{INTRODUCCIÓN}

En el año 2000, el Parlamento Europeo aprobó la Directiva Marco de Aguas (en adelante DMA) (Directiva 60/2000), una de las directivas más importantes en materia ambiental de las últimas décadas, en la que se establece el marco comunitario de actuación en materia de aguas, prestando especial atención a aspectos administrativos, ambientales, económicos y de participación ciudadana. Su aprobación ha supuesto para los Estados Miembros la obligación de realizar importantes cambios en sus sistemas de planificación y en sus organismos de gestión de los recursos hídricos (Andersson et al, 2012: 75; Junier and Mostert, 2012: 3; Orsted et al, 2013: 438), que aún no han finalizado. En el caso de España, ha sido necesario modificar las unidades de gestión preestablecidas, reunificando organismos de cuenca preexistentes y creando otros nuevos.

Entre las novedades que introduce la DMA se encuentra la necesidad de incrementar la «integración de la protección y la gestión sostenible del agua en otros ámbitos políticos comunitarios, tales como las políticas en materia de energía, transporte, agricultura, pesca, política regional y turismo» (Consideración 16). Esta cuestión no vuelve a aparecer de forma explícita en el resto del articulado aunque si implícitamente, especialmente la relación que debe propiciarse entre la planificación/gestión del agua y la planificación espacial. Los objetivos que plantea la Directiva son inasumibles desde una planificación/gestión sectorial del agua por lo que la integración de esta política sectorial con la territorial se acepta como necesaria, sin embargo en el texto normativo no se especifica en qué debe consistir dicha integración, ni cuál es la mejor forma para lograrla.

Este argumento acerca de la necesidad de integrar las políticas territoriales e hidrológicas está ampliamente aceptado en la cultura científica actual. Desde la década de los 90 se registra un incremento importante en el número de trabajos que abordan estas cuestiones, aunque es posible encontrar trabajos científicos y normativos mucho más antiguos en los que se maneja la idea de gestión conjunta de recursos territoriales, hídricos y ambientales, especialmente en el contexto de las cuencas hidrográficas (Real Decreto de 5 de marzo de 1926; White, 1957; Ingram, 1973; Schramm, 1980; Lundquist et al, 1985). La procedencia de los trabajos recientes es muy dispar, no sólo europea: Alemania (Moss, 2004: 85); Australia (Johnson et al, 1996: 303); Canadá (Carter et al 2005: 115); España (Del Moral, 2006: 30); Estados Unidos (Mitchell, 1990: 4); Holanda (Woltjer and Al, 2007: 211; Wiering and Immink, 2006: 423); Israel (Carmon and Shamir, 2010: 181); Reino Unido (Kidd and Shaw, 2007: 319). Está generalizado el reconocimiento de la integración como mecanismo que puede contribuir a reducir los conflictos actuales y futuros vinculados con el agua y el territorio, posibilitando un mejor desarrollo territorial desde la búsqueda de equilibrios entre lo ambiental, lo social y lo económico, pero las aportaciones de carácter operativo son muy escasas. Este déficit de sugerencias metodológicas aplicables, se produce por la complejidad del objetivo y también por la falta de consenso y precisión acerca de qué es la integración (Biswas, 2004:251; García, 2008: 23), cuáles son sus objetivos específicos, cómo se puede lograr, cómo se puede medir, qué efectos puede tener en cuestiones económicas y sociales... Entre la literatura existente sí cabe reseñar algunos trabajos que:

- Identifican cuestiones que deben incorporarse en los documentos de planificación (Del Moral 2006, 2009; Hedelin 2007) 
- Plantean cuestiones que deben afrontarse en la gobernanza de la gestión integrada de recursos hídricos (Jonch-Clausen and Fugl, 2001; Kidd and Shaw, 2007)

- Examinan diferentes modelos de gobernanza (Woltjer and Al, 2007; Kidd and Shaw, 2007; Orsted et al, 2013)

- Proponen instrumentos para valorar el nivel de integración (Carter et al, 2005; Hedelin, 2008; Plummer et al, 2011; Andersson et al, 2012)

Entre los trabajos publicados hay algunos (Del Moral, 2009; Carter, 2007) que le otorgan a los documentos de planificación un papel muy destacado en una primera fase hacia la integración. En los planes territoriales se puede incorporar el enfoque hidrológico y viceversa (el enfoque territorial en los planes hidrológicos), de manera que, manteniendo las dos estructuras sectoriales de planificación y gestión, los instrumentos vayan retroalimentándose y avanzando hacia una gestión integrada, mitigando los conflictos y fomentando las sinergias.

En el contexto europeo, esta idea de incluir la perspectiva hidrológica en los planes territoriales a la que se alude en el párrafo precedente, supone obligatoriamente la incorporación de los objetivos y medidas de la referida DMA. La aplicación de la Directiva a través de los planes hidrológicos de cuenca puede desencadenar procesos espaciales, ambientales y económicos muy relevantes para la gestión integrada de los recursos hídricos en los ámbitos territoriales de planificación. Los planes territoriales deben contemplar estas posibles transformaciones para poder gestionar los impactos asociados y que no se restrinja el desarrollo de actividades y oportunidades futuras (Carter, 2007: 332). Se trata por tanto de afianzar la integración entre los instrumentos de planificación de naturaleza distinta, los territoriales y los hidrológicos.

En España los planes hidrológicos relativos al primer ciclo de planificación reconocido en la DMA se han publicado con retraso. En la Directiva se fijaba el 2009 como fecha límite para su aprobación y en nuestro país, como se puede apreciar en la Figura 1, por motivos diversos (cambios en las unidades de gestión, en las administraciones competentes, arraigo de políticas clásicas, aprobación tardía del reglamento que adapta los mecanismos de planificación- RD 907/2007) no se ha cumplido para ninguna Demarcación.

Este hecho, además de acarrear una sanción económica (Sentencia del Tribunal de Justicia de la Unión Europea de 4 de octubre de 2012) y de provocar el incumplimiento de los objetivos esperados en este ciclo de planificación (FNCA, 2014) dificulta la medida de la integración entre instrumentos de planificación territorial e hidrológica.

Por ello, este artículo siguiendo la línea de los trabajos que proponen instrumentos para valorar la magnitud de la integración (Carter et al, 2005; Hedelin, 2008; Plummer et al, 2011) ha optado por proponer una metodología sencilla para evaluar el grado de adaptación de los planes territoriales a la DMA, como primera aproximación a la valoración de la integración.

El artículo se estructura en dos grandes bloques. En el primero se determinan cuáles son las implicaciones territoriales más relevantes que puede tener la DMA (a través del análisis de sus principios, objetivos y medidas) y se identifican criterios para la valoración de la adaptación de los planes territoriales a la DMA. El segundo bloque presenta los resultados de la aplicación del método de evaluación propuesto en cinco de los planes territoriales de escala subregional (POT, en adelante) aprobados en Andalucía, concluyendo con la discusión de los mismos. 


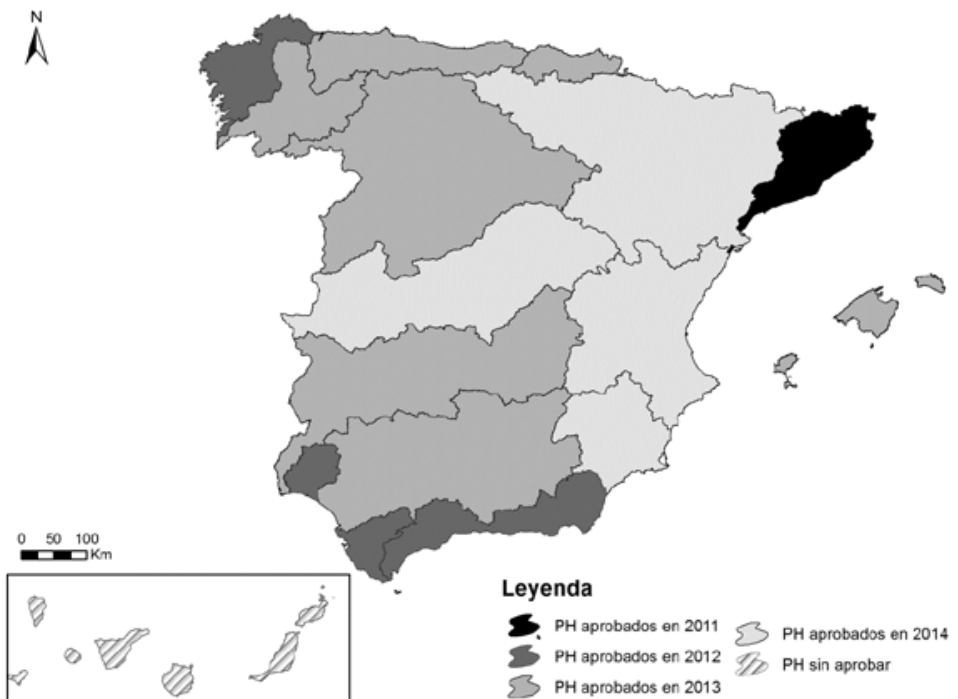

Fuente: elaboración propia a partir del BOE.

\section{UN MÉTODO PARA VALORAR LA ADAPTACIÓN DE LOS PLANES TERRITORIALES A LA DMA A TRAVÉS DE CRITERIOS}

La identificación de criterios que ayuden a evaluar de manera sistemática algunos aspectos de las figuras de planificación se ha empleado en varios trabajos en el contexto de la integración de los recursos hídricos (Carter et al, 2005; Hedelin, 2007; Valenzuela y Matarán, 2008; Del Moral, 2009; Plummer et al, 2011), aunque en otros ámbitos disciplinares existen multitud de ejemplos (Baer, 1997; Pettit and Pullar, 1999; Laurian et al, 2004). Esto se debe a que la definición de criterios evaluadores comporta varias ventajas: (1) en primer lugar se facilita la evaluación, al sistematizar el trabajo de revisión y análisis de los planes, que suele ser laborioso; (2) en segundo lugar, constituyen una herramienta para poner de relieve recomendaciones prácticas (Hedelin, 2008: 239), al señalar en que aspectos existen deficiencias; (3) y en último lugar, aunque no menos importante, los criterios identificados pueden utilizarse para ir monitorizando los avances de los planes, pues es importante pensar en los procesos de planificación como procesos cíclicos y de mejora continua más que como simples actos de planificación (Allan, 2012: 172).

En este artículo los criterios que se van a definir posibilitan la evaluación del grado de adaptación que presentan los planes territoriales a la DMA. La definición de estos criterios se realiza en la segunda de las tres fases que constituyen la secuencia metodológica completa:

1. Identificación de las principales implicaciones territoriales de la DMA y su normativa derivada en España 
2. Definición de criterios para valorar la adaptación de los planes territoriales a la DMA

3. Valoración de la adaptación de los planes territoriales a la DMA

\section{II.1. Identificación de las principales implicaciones territoriales de la DMA y su normativa deri- vada en España}

Como se ha señalado, la amplitud de cambios introducidos por la DMA ha modificado todo el marco contextual de la política de aguas a nivel europeo con consecuencias en otras muchas políticas y a diferente escala. Las medidas que se proponen en la propia DMA y en los documentos normativos estatales y autonómicos derivados de ella, así como sus potenciales consecuencias sobre el territorio serán las cuestiones que los planes territoriales deberán asumir, si se apuesta por la integración como mecanismo tanto de reducción de los conflictos actuales y futuros como de armonización entre los instrumentos de planificación de las dos políticas.

En este artículo, además de la DMA se han analizado, como normativa de menor rango que sigue sus principios y que es de aplicación en los ámbitos de estudio seleccionados: el Reglamento de Planificación Hidrológica (RD 907/2007) (RPH2007, en adelante); y la Ley de Aguas de Andalucía (Ley 9/2010) (LAA2010, en adelante).

La identificación de las principales implicaciones territoriales se realiza a través de un proceso lineal que comienza con el reconocimiento de los principios y los objetivos generales de la DMA, para posteriormente identificar cuáles son las medidas que ayudan a lograrlos y que pueden tener mayores consecuencias territoriales.

Los principios fundamentales que guían el contexto actual en materia de agua son (GEA y Arrojo, 2008: 5; Valenzuela et Rigosi, 2009: 148; Collins et al, 2012: 50): principio de sostenibilidad; principios de racionalidad económica y recuperación de costes; principio de precaución/prevención; principio de participación y transparencia; principio de integración.

A partir de estos cinco principios fundamentales se pueden estructurar los objetivos principales de la DMA y precisar algunas de las medidas propuestas con mayor potencial para generar transformaciones territoriales.

Para clarificar todo este proceso aparece a continuación una tabla (Tabla 1) con los principios enunciados, junto con los objetivos y las medidas que hemos considerado y las principales consecuencias territoriales que creemos pueden originarse en el contexto español (se han concebido según las condiciones particulares de nuestro país). En la tabla también se identifican para cada una de las medidas los artículos de la DMA, del RPH2007 y de la LAA2010 en los que se alude a ellas.

En la tabla aparecen algunas cuestiones que conviene matizar:

- El mantenimiento de caudales ecológicos es la primera de las medidas registrada en la tabla para cumplir con el objetivo de prevención del deterioro y mejora del estado cuantitativo de las masas de agua, a pesar de no aparecer de manera explícita en la DMA. Sin embargo, debido a los objetivos de calidad y cantidad que establece para todas las masas de agua de la Unión Europea, es indudable la necesidad de mantener unos caudales mínimos que permitan el funcionamiento del ecosistema. Así se ha interpretado en las normativas derivadas de los Estados Miembros y también en 
Tabla 1

PRINCIPIOS, OBJETIVOS Y MEDIDAS E IMPLICACIONES TERRITORIALES DE LADMA Y DE SU NORMATIVA DERIVADA EN ESPAÑA

\begin{tabular}{|c|c|c|c|c|c|c|}
\hline \multirow{2}{*}{$\begin{array}{l}\text { Principios } \\
\text { DMA }\end{array}$} & \multirow[b]{2}{*}{$\begin{array}{l}\text { Objetivos } \\
\text { DMA }\end{array}$} & \multirow[b]{2}{*}{$\begin{array}{l}\text { Medidas con capacidad para } \\
\text { influir en el territorio }\end{array}$} & \multicolumn{3}{|c|}{ Artículos Normativa de aguas } & \multirow[b]{2}{*}{ Principales implicaciones a nivel territorial } \\
\hline & & & DMA & $\begin{array}{l}\text { RPH } \\
2007\end{array}$ & $\begin{array}{l}\text { LAA } \\
2010\end{array}$ & \\
\hline \multirow{15}{*}{ 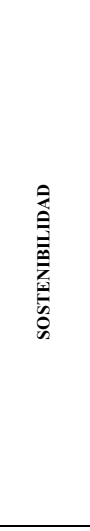 } & \multirow{10}{*}{$\begin{array}{c}\text { Prevenir el } \\
\text { deterioro y } \\
\text { mejorar el estado } \\
\text { CUANTITATIVO } \\
\text { de las masas de } \\
\text { agua }\end{array}$} & \multirow{10}{*}{$\begin{array}{l}\text { - Mantenimiento de Caudales } \\
\text { ecológicos } \\
\text { - Mantenimiento equilibrios } \\
\text { - Recursos hídricos- } \\
\text { consumos } \\
\text { - Límites demandas } \\
\text { - Nuevos recursos } \\
\text { - Ahorro } \\
\text { Extracción- } \\
\text { alimentación acuíferos } \\
\text { - Recarga acuíferos } \\
\text { - Limites } \\
\text { concesionales } \\
\text { - Revisión concesional }\end{array}$} & & $17 ; 18$ & 44 & \multirow{10}{*}{$\begin{array}{l}\text { CAMBIOS EN LOS CULTIVOS } \\
\text { MODIFICACIONES EN LOS } \\
\text { SISTEMAS DE RIEGO } \\
\text { TRANSFORMACIONES USOS DEL } \\
\text { SUELO (EMPLAZAMIENTO } \\
\text { SUPERFICIE) } \\
\text { ABANDONO } \\
\text { AGRÍCOLAS } \\
\text { DESTRICCIONES } \\
\text { DESARROLLO URBANÍSTICO AL }\end{array}$} \\
\hline & & & & & & \\
\hline & & & & & & \\
\hline & & & & 48 & & \\
\hline & & & & 60 & 46 & \\
\hline & & & & $14 ; 47$ & $7 ; 25 ; 5$ & \\
\hline & & & $4 ; 11$ & $35 ; 54$ & 54 & \\
\hline & & & 11 & 53 & 56 & \\
\hline & & & & 24 & $45 ; 51$ & \\
\hline & & & & $14 ; 47$ & $44 ; 45$ & \\
\hline & \multirow{5}{*}{$\begin{array}{c}\text { Prevenir el } \\
\text { deterioro y } \\
\text { mejorar el estado } \\
\text { CUALITATIVO } \\
\text { de las masas de } \\
\text { agua }\end{array}$} & \multirow{5}{*}{$\begin{array}{l}\text { - Implantación de mecanismos } \\
\text { para el control de la } \\
\text { contaminación } \\
\text { Depuración } \\
\text { - Límite productos } \\
\text { contaminantes } \\
\text { - Protección y conservación } \\
\text { de cauces } \\
\text { Zonas y perímetros de } \\
\text { protección }\end{array}$} & & & & \multirow{5}{*}{$\begin{array}{l}>\text { IMPLANTACIÓN SISTEMAS DE } \\
\text { DEPURACIÓN } \\
\text { RESTRICCIONES ESPACIALES A } \\
\text { DETERMINADOS USOS DEL SUELO }\end{array}$} \\
\hline & & & 7 & $14 ; 45$ & $11 ; 14 ; 25 ; 34$ & \\
\hline & & & $\begin{array}{l}4 ; 10 \\
11 ; 16 \\
\end{array}$ & $\begin{array}{c}35 ; \\
49 ; 51 ; 58 \\
\end{array}$ & $13 ; 55$ & \\
\hline & & & & & $21 ; 40$ & \\
\hline & & & 7 & $23 ; 24 ; 35 ; 57$ & $40 ; 55$ & \\
\hline \multirow{3}{*}{ 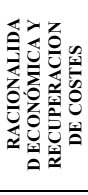 } & \multirow{3}{*}{$\begin{array}{l}\text { Incentivar el uso } \\
\text { eficiente de los } \\
\text { recursos hídricos } \\
\text { Recuperar los } \\
\text { costes de los } \\
\text { servicios } \\
\text { relacionados con } \\
\text { el agua } \\
\end{array}$} & \multirow{3}{*}{$\begin{array}{l}\text { - Aplicación de tarifas para el } \\
\text { regadío } € / m 3 \\
\text { - Incremento del precio del agua }\end{array}$} & & 47 & & \multirow{3}{*}{$\begin{array}{l}>\text { MODIFICACIONES EN } \\
\text { SISTEMAS DE RIEGO } \\
\text { TRANSFORMACIONES USOS } \\
\text { SUELO (EMPLAZAMIENTO Y Y } \\
\text { SUPERFICIE) } \\
>\text { ABANDONO } \\
\text { AGRÍCOLAS }\end{array}$} \\
\hline & & & 9 & $46 ; 47$ & 98 & \\
\hline & & & & $42 ; 46$ & $\begin{array}{c}73 ; 79 ; 91 ; 97 \\
100\end{array}$ & \\
\hline \multirow{5}{*}{ 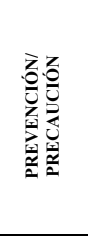 } & \multirow{4}{*}{$\begin{array}{l}\text { Prevención y } \\
\text { gestión de riesgos } \\
\text { de inundación }\end{array}$} & \multirow{4}{*}{$\begin{array}{l}\text { - Mantenimiento/Restauración } \\
\text { de cauces } \\
\text { - Actuaciones hidrológico- } \\
\text { forestales } \\
\text { - Actuaciones de protección } \\
\text { - Perímetros de protección } \\
\text { (limitación usos del suelo y } \\
\text { actividades) }\end{array}$} & & & $26 ; 40$ & \multirow{4}{*}{$\begin{array}{l}\text { RESTRICCIONES ESPACIALES A } \\
\text { DETERMINADOS USOS DEL SUELO } \\
>\text { IMPACTOS D DE } \\
\text { INFRAESTRUCTURAS } \\
\text { PROTECCIÓN) } \\
>\text { MEJORA FORESTAL } \\
>\text { MEJORA RIBERA DE LOS CURSOS } \\
\text { DE AGUA }\end{array}$} \\
\hline & & & & & 29 & \\
\hline & & & & 59 & 29 & \\
\hline & & & & & $11 ; 41 ; 60$ & \\
\hline & Gestión de sequías & $\begin{array}{l}\text { - Limites extraordinarios de } \\
\text { demandas y caudales } \\
\text { ecológicos }\end{array}$ & & 18 & 63 & $\begin{array}{l}\text { CAMBIOS EN LOS CULTIVOS } \\
\text { ABANDONO } \\
\text { AGRÍCOLAS }\end{array}$ \\
\hline \multirow{2}{*}{ 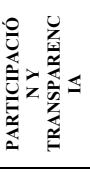 } & \multirow{2}{*}{$\begin{array}{l}\text { Incorporar la } \\
\text { opinión de todas } \\
\text { las partes } \\
\text { interesadas } \\
\text { Inclusión de "el } \\
\text { conocimiento } \\
\text { local" } \\
\end{array}$} & \multirow[t]{2}{*}{$\begin{array}{l}\text { - Procedimientos que permitan } \\
\text { participación activa }\end{array}$} & 14 & $71 ; 72 ; 74 ; 75$ & $17 ; 18 ; 20 ; 62$ & \multirow{5}{*}{$\begin{array}{l}l \\
\\
\\
\text { A PARTIR DE ESTE PRINCIPIO SE } \\
\text { PUEDE ENTENDER LA NECESIDAD } \\
\text { DE INCORPORAR EN LA } \\
\text { PLANIFICACIÓN TERRITORIAL LOS } \\
\text { OBJETIVOS Y MEDIDAS DE LA DMA } \\
\text { (COMO SOSTIENE ESTE TRABAJO). } \\
\text { LAS IMPLICACIONES SERÍAN } \\
\text { TODAS LAS IDENTIFICADAS EN LAS } \\
\text { FILAS ANTERIORES } \\
\end{array}$} \\
\hline & & & $15 ; 18$ & $65 ; 73 ; 79 ; 80$ & 19 & \\
\hline \multirow{3}{*}{ 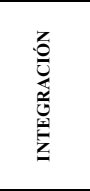 } & \multirow{3}{*}{$\begin{array}{l}\text { Ajustar la gestión } \\
\text { de los recursos } \\
\text { hídricos a las } \\
\text { características de } \\
\text { cada territorio } \\
\text { Mantener la } \\
\text { unidad de cuenca }\end{array}$} & \multirow{3}{*}{$\begin{array}{l}\text { - Incremento de las relaciones } \\
\text { entre Administraciones } \\
\text { - Mejora del ajuste entre } \\
\text { documentos de planificación } \\
\text { territorial e hidrológica } \\
\text { - Planes hidrológicos de Cuenca }\end{array}$} & & 71 & $20 ; 29$ & \\
\hline & & & & 71 & 42 & \\
\hline & & & 13 & 2 & 3 & \\
\hline
\end{tabular}

Fuente: elaboración propia a partir de la DMA (Directiva 60/2000), Real Decreto 907/2007 y Ley 9/2010.

España donde los problemas vinculados a la cantidad de recursos hídricos son más frecuentes. Las repercusiones territoriales que se derivan de esta medida aparecen al considerarse los caudales ecológicos como «una restricción que se impone con carácter general a los sistemas de explotación» (Art. 17 y 18 Reglamento de Planificación Hidrológica (RD 907/2007); art. 44 Ley de Aguas de Andalucía (Ley 9/2010). 
- La aplicación de cualquiera de las tres medidas señaladas para el objetivo de prevención del deterioro y mejora del estado cuantitativo de las masas de agua, supone una reducción del volumen de recursos hídricos disponibles para los diferentes usos del agua (agricultura de regadío, turismo, nuevos desarrollos urbanos, industria, sector energético...). Sin embargo, al existir una regla de prioridad para el uso del agua destinado a abastecimiento de población (Art. 60, Real Decreto Legislativo $1 / 2001$ ), el reajuste de los volúmenes disponibles tendrá las mayores repercusiones en el regadío.

- En España las medidas «que incentiven el uso eficiente del agua» pasan irremediablemente por un incremento en el precio del agua que tendrán un gran impacto especialmente en la agricultura ya que tradicionalmente este ha sido un recurso fuertemente subvencionado (Valera-Ortega et al, 1998: 194; Sáenz, 2002: 200; Gómez et al, 2008: 17). Además, en muchas zonas de riego es preciso cambiar el sistema de tarificación pasando de $€ / \mathrm{m}^{2} \mathrm{a} € / \mathrm{m}^{3}$, de manera que se pague por el volumen realmente consumido en cada parcela (Castillo y González, 2002, p. 266; Rico, 2006, p. 120).

- Dentro del principio de «prevención/precaución», para las sequías sólo se ha incluido una medida para la gestión, ya que las medidas para la prevención serían las mismas que las registradas para cumplir con el principio de Sostenibilidad. Si existe un buen estado de todas las masas de agua desde el punto de vista de la calidad y la cantidad se estarán previniendo los efectos de las sequías y sólo quedará establecer medidas puntuales a aplicar en los periodos en que, debido a las características propias del clima mediterráneo, aparece de manera natural este fenómeno. En este sentido, a través de la Ley 10/2001 del Plan Hidrológico Nacional se reguló en España la obligatoriedad de que los organismos de cuenca aprobaran Planes especiales de actuación en situaciones de alerta y eventual sequía, y cuyas determinaciones también deberán ser asumidas en los planes territoriales. El principio de integración es el que fundamenta el presente trabajo y las medidas identificadas para alcanzar los objetivos que se derivan de él supondrán la incorporación en los documentos de planificación territorial de todos los principios, objetivos y medidas de la DMA recogidos en el Tabla 1. Por ello, las implicaciones territoriales del principio de integración serían todas las que aparecen en dicha tabla.

\section{II.2. Definición de criterios evaluadores para valorar la adaptación de los planes territoriales a la DMA}

En la tabla que aparece a continuación (Tabla 2), se recogen de nuevo las medidas de la DMA así como las potenciales consecuencias que se han detallado en el Tabla 1, que sirven para definir los 10 criterios que posibilitan la valoración de la adaptación de los planes territoriales a la DMA.

Los criterios evaluadores propuestos se pueden agrupar en tres bloques según la intención con la que se formulan:

1. Criterios estructurales: persiguen descubrir si los planes territoriales han asumido ciertas condiciones esenciales relativas a los recursos hídricos derivadas de la DMA 


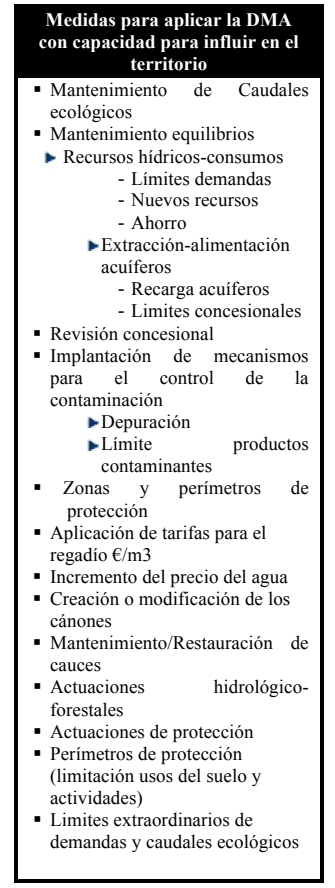

Fuente: elaboración propia.

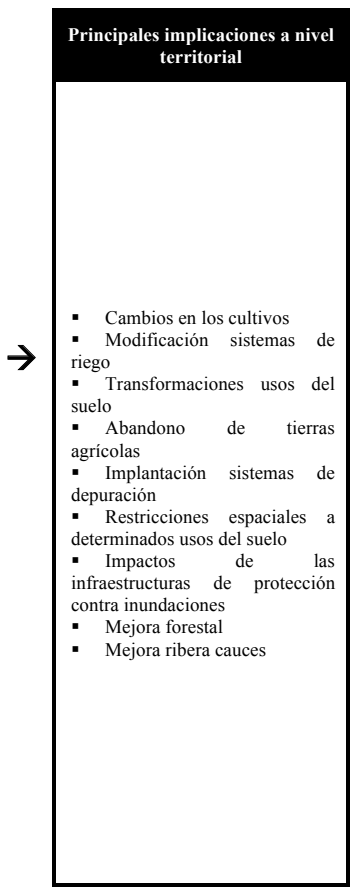

(criterios 1, 2 y 3). El criterio 1 persigue revelar si los planes territoriales reconocen la importancia de los caudales ecológicos y la repercusión que pueden tener a nivel territorial al limitar la disponibilidad de recursos hídricos para los diferentes usos. Los criterios 2 y 3 hacen alusión a la necesidad de mantener el equilibrio entre los recursos hídricos disponibles y los consumos, así como entre la extracción y la alimentación de los acuíferos, respectivamente.

2. Criterios propositivos: están orientados a desvelar si los planes territoriales incorporan medidas concretas que pueden ayudar a conseguir los objetivos de la DMA (criterios $4,5,6,7$ y 8 ).

3. Criterios adaptativos: intentan poner de manifiesto si los planes territoriales tienen capacidad de respuesta para los cambios territoriales principales que puede ocasionar la DMA (criterio 9 y 10). El criterio 9 persigue descubrir si los planes podrán adaptarse a las consecuencias principales que la DMA pueden provocar en la agricultura de regadío mientras que el criterio 10 pondrá de relieve el grado de conexión entre los planes territoriales y las leyes y normas de protección y gestión de los recursos hídricos. 


\section{II.3. Valoración de la adaptación de los planes territoriales a la DMA}

Esta tercera fase se puede dividir a su vez en dos. En primer lugar habrá que seleccionar los planes que se van a evaluar y posteriormente realizar la valoración.

\section{II.3.1. Selección de los planes a evaluar}

La metodología que aquí se propone podría aplicarse a planes territoriales de cualquier escala aunque los planes de escala subregional pueden constituir laboratorios apropiados. La naturaleza y la escala de estos planes hacen que puedan emplearse como nexo entre los planes hidrológicos de cuenca y los planes urbanísticos de escala municipal, y por tanto valorar su adaptación a la DMA y trabajar para mejorarla puede suponer un hito importante en el camino hacia la gestión integrada del agua y del territorio.

En este trabajo se han elegido cinco planes cuyos ámbitos de aplicación se encuentran en Andalucía y que se rigen por la Ley 1/1994 de Ordenación del Territorio de la dicha Comunidad Autónoma: POT Bahía de Cádiz (2004); POT Litoral Occidental de Huelva (2006); POT Levante de Almería (2009); POT Costa Noroeste de Cádiz (2011); POT Costa Tropical de Granada (2012). La característica de que los planes a valorar se aprobaran en distinta fecha da la oportunidad de descubrir si con el paso de los años y la entrada en vigor de nueva normativa derivada de la DMA (RD 907/2007; Ley 9/2010) se aprecian cambios en los resultados.

\section{II.3.2. Elementos de valoración: número de referencias y tratamiento normativo}

El valor de la adaptación de los planes territoriales a la DMA será dependiente del número de referencias que existan en los documentos normativos de los planes para cada criterio y del tratamiento normativo que tengan.

A través de la lectura sistemática de la normativa de los planes se irán extrayendo las referencias que den respuesta a los 10 criterios evaluadores que posteriormente se contabilizaran. El número total no va a corresponder necesariamente con el número de artículos de la normativa donde se hace alusión a cada uno de los criterios, ya que cuando los artículos son extensos y presentan diferentes apartados, se contabiliza a cada uno de ellos como una referencia independiente. Asimismo, hay referencias que al hacer alusión a varias cuestiones o recopilar medidas con diferente objetivo se registran en más de un criterio.

La posibilidad de identificar el tratamiento normativo con que aparecen las referencias depende de las características que posean los planes que se van a evaluar. Los cinco planes seleccionados en este artículo distinguen entre «determinación con carácter de Norma, Directriz o Recomendación», en cumplimiento del artículo 21 de la Ley 1/1994 de Ordenación del Territorio de Andalucía por la que se rigen:

- Normas: «determinaciones de aplicación directa vinculante para las Administraciones Públicas y para los particulares, en los suelos urbanizables y no urbanizables».

- Directrices: «determinaciones vinculantes en cuanto a sus fines. Con sujeción a ellas, los órganos competentes de las Administraciones públicas a quién corresponda su aplicación establecerán las medidas concretas para la consecución de dichos fines». 
- Recomendaciones: «determinaciones de carácter indicativo dirigidas a las Administraciones Públicas que, en caso de apartarse de las mismas, deberán justificar de forma expresa la decisión adoptada y su compatibilidad con los objetivos de la Ordenación del Territorio».

A partir de estos dos elementos de valoración el resultado será más positivo, es decir el plan estará mejor adaptado a la DMA, cuando se identifiquen referencias para un mayor número de criterios evaluadores, existan varias referencias para cada criterio y las referencias tengan un tratamiento normativo más operativo. Los planes que contemplen referencias con «carácter de norma» están obligados a aplicarlas y por lo tanto incidirán en una mejora de la perspectiva hidrológica con la que se gestiona el territorio mientras que las referencias con «carácter de recomendación» pueden quedar sólo en indicaciones sobre el papel.

Toda la información que se recopile de los planes en esta fase se puede sintetizar en una tabla en la que se registra el número de referencias encontradas para cada criterio evaluador y el «carácter» con el que se presentan en la normativa del POT (ver Tabla 4, en el epígrafe «Resultados» que aparece más adelante).

\section{APLICACIÓN DE LA METODOLOGÍA DE EVALUACIÓN}

\section{III.1. Los planes territoriales subregionales de Andalucía}

En la comunidad autónoma andaluza existen dos instrumentos de planificación integral del territorio definidos en la Ley 1/1994, de Ordenación del Territorio de la Comunidad Autónoma de Andalucía: el Plan de Ordenación del Territorio de Andalucía (POTA, en adelante) (Decreto 206/2006) y los Planes de Ordenación del Territorio de ámbito subregional (POT, en adelante).

El POTA determina los elementos básicos de la organización y estructura del territorio de Andalucía, siendo el marco de referencia territorial para los demás planes y para la acción pública en general.

Los POTs establecen los elementos básicos para la organización y estructura del territorio en su ámbito, siendo el marco de referencia territorial para el desarrollo y coordinación de las políticas, planes, programas y proyectos de las Administraciones y Entidades Públicas así como para las actividades de los particulares. El ámbito de cada plan abarca el conjunto de términos municipales completos y contiguos, que por sus características físicas, funcionales y socioeconómicas conformen un área coherente de planificación territorial (artículo 14 de la Ley 1/1994).

Actualmente existen 17 POTs aprobados, el de la Aglomeración Urbana de Granada es el más antiguo (aprobado en el año 2000) y el del Sur de Córdoba el más reciente (aprobado en el año 2012). La superficie afectada por estos instrumentos de planificación territorial supone el 29,7\% de la superficie total de la Comunidad Autónoma.

Para aplicar la metodología propuesta en el presente artículo la selección de los planes se ha realizado en base a su fecha de aprobación ya que el resultado de la valoración podría verse afectado por los cambios que se han producido en el marco normativo desde la entrada en vigor de la DMA. En la siguiente figura (Figura 2) se han registrado las fechas de los 
principales hitos normativos en materia de aguas de nuestro país desde el año 2000 (año de entrada en vigor de la DMA) y la fecha de aprobación de los POTs en Andalucía. Se ha elegido, desde la trasposición de la DMA al derecho español (año 2003), un POTs de cada una de las distintas fechas de aprobación (los elegidos aparecen sombreados en la Figura 2): POT Bahía de Cádiz (2004); POT Litoral Occidental Huelva (2006); POT Levante de Almería (2009); POT Costa Noroeste de Cádiz (2011); POT Costa Tropical de Granada (2012). Al elegir estos cinco planes, como se apreciaba en la epígrafe anterior, además de evaluar la adaptación de cada uno de ellos a la DMA podemos descubrir si con el paso de los años, la entrada en vigor de nueva normativa y el afianzamiento de los principios y objetivos de la DMA, se producen avances o no se aprecia ninguna mejora.

Figura 2

FECHAS DE APROBACIÓN DE LA NORMATIVA DE AGUAS Y LOS POTS ANDALUCES

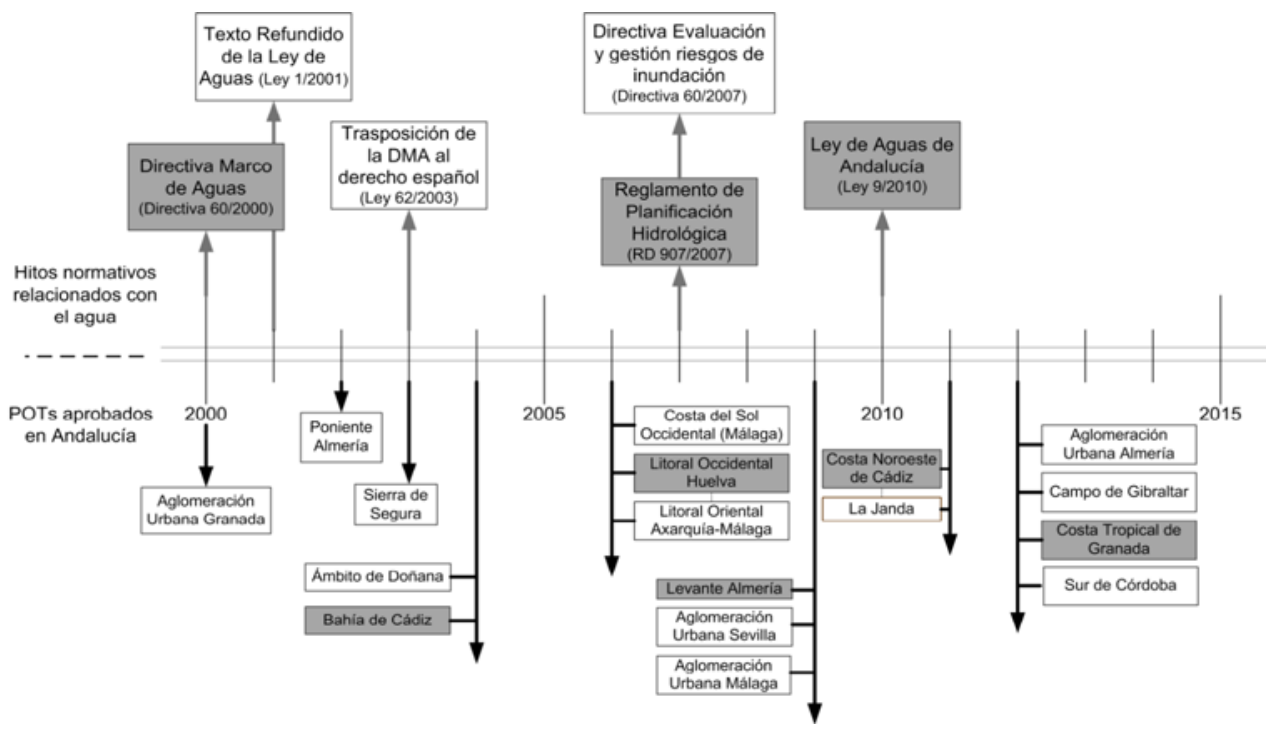

Fuente: elaboración propia.

A continuación aparece un mapa de Andalucía (Figura 3) en el que se señalan los ámbitos de los planes territoriales de escala subregional que se encuentran aprobados y se remarcan los que se han elegido como ámbitos de estudio para este artículo.

Para contextualizar los ámbitos cuyos planes se van a evaluar se presenta a continuación una tabla (Tabla 3) con el número de municipios que integran el ámbito de aplicación del POT, la población que reside en el ámbito (año 2010), la superficie (en $\mathrm{km}^{2}$ ) y la densidad de población. También es interesante resaltar que todos los ámbitos subregionales elegidos poseen gran parte de sus ámbitos localizados en las demarcaciones hidrográficas intracomunitarias de Andalucía (Demarcación Hidrográfica de las Cuencas Mediterráneas Andaluzas; Demarcación Hidrográfica Tinto, Odiel y Piedras; Demarcación Hidrográfica GuadaleteBarbate) siendo la administración competente es también la autonómica. 

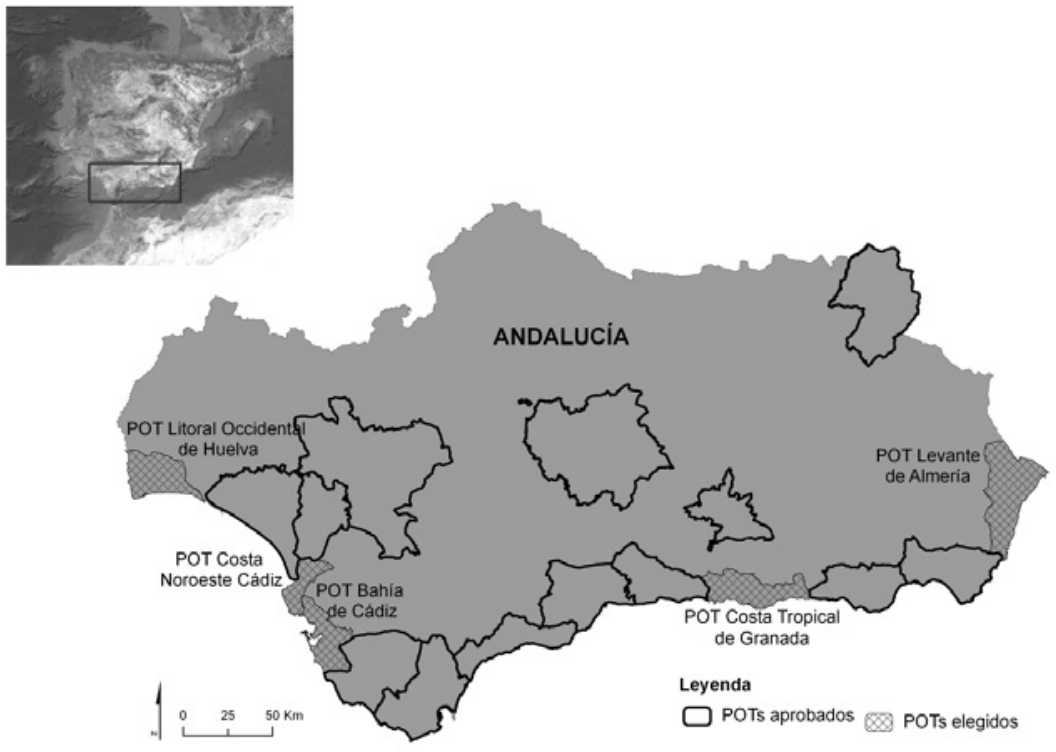

Fuente: elaboración propia.

Tabla 3

DATOS RELEVANTES DE LOS POTS EN LOS QUE SE VAA VALORAR EL GRADO DEADAPTACIÓN A LA DMA

\begin{tabular}{|c|r|r|r|r|r|l|}
\hline Bahía de Cádiz & 2004 & 5 & 430.276 & 606,1 & 709,9 & - Guadalete-Barbate \\
\hline Litoral Occidental de Huelva & 2006 & 7 & 105.763 & 732,1 & 144,46 & $\begin{array}{l}\text { - Tinto, Odiel y Piedras } \\
\text { - Guadiana }\end{array}$ \\
\hline Levante de Almería & 2009 & 11 & 90.325 & $1.198,8$ & 75,35 & $\begin{array}{l}\text { - Cuencas Mediterráneas } \\
\text { Andaluzas } \\
\text { - Segura }\end{array}$ \\
\hline Costa Noroeste de Cádiz & 2011 & 4 & 121.201 & 360,7 & 336,01 & $\begin{array}{l}\text { - Guadalete-Barbate } \\
\text { - Guadalquivir }\end{array}$ \\
\hline Costa Tropical de Granada & 2012 & 17 & 127.613 & 786,7 & 162,21 & $\begin{array}{l}\text { - Cuencas Mediterráneas } \\
\text { Andaluzas }\end{array}$ \\
\hline
\end{tabular}

Fuente: elaboración propia a partir de datos de la Consejería de Obras Públicas y Viviendas de la Junta de Andalucía.

\section{III.2. Resultados}

Este apartado presenta los resultados obtenidos al aplicar la metodología propuesta a los cinco planes subregionales de Andalucía seleccionados. Como se establece en dicha metodología se han revisado los documentos normativos y se ha registrado en la tabla siguiente (Tabla 4) el número de referencias encontradas para cada criterio según el «carácter» con el que aparecen. 
Tabla 4

RESULTADOS DEL ANÁLISIS DE LOS POTS PARA EVALUAR SU GRADO DE ADAPTACIÓN A LA DMA (NÚMERO DE REFERENCIAS IDENTIFICADAS)

\begin{tabular}{|c|c|c|c|c|c|c|c|c|c|c|c|c|c|c|c|}
\hline \multirow[t]{2}{*}{ Criterios evaluadores de la adaptación a la DMA } & \multicolumn{3}{|c|}{$\begin{array}{l}\text { POT Bahía de } \\
\text { Cádiz }\end{array}$} & \multicolumn{3}{|c|}{$\begin{array}{l}\text { POT Lit. } \\
\text { Occid. Huelva }\end{array}$} & \multicolumn{3}{|c|}{$\begin{array}{l}\text { POT Levante } \\
\text { Almería }\end{array}$} & \multicolumn{3}{|c|}{$\begin{array}{l}\text { POT Costa } \\
\text { Noreste Cádiz }\end{array}$} & \multicolumn{3}{|c|}{$\begin{array}{l}\text { POT Costa } \\
\text { Tropical Gra- } \\
\text { nada }\end{array}$} \\
\hline & $\mathrm{N}$ & $\mathrm{D}$ & $\mathrm{R}$ & $\mathrm{N}$ & $\mathrm{D}$ & $\mathrm{R}$ & $\mathrm{N}$ & $\mathrm{D}$ & $\bar{R}$ & $\mathrm{~N}$ & $\mathrm{D}$ & $\mathrm{R}$ & $\mathrm{N}$ & $\mathrm{D}$ & $\mathrm{R}$ \\
\hline \multicolumn{16}{|l|}{\begin{tabular}{l|l} 
1. Reconoce los caudales ecológicos como restricciones a & \\
los recursos hídricos del ámbito &
\end{tabular}} \\
\hline $\begin{array}{l}\text { 2. Reconoce la necesidad de mantener el equilibrio entre } \\
\text { los recursos hídricos y los consumos }\end{array}$ & & 1 & & 1 & & & & 4 & & 1 & 1 & & 2 & 2 & \\
\hline $\begin{array}{l}\text { 3. Reconoce la necesidad de mantener el equilibrio entre } \\
\text { extracción y alimentación de acuíferos }\end{array}$ & & & & & & & & & & 1 & & & & & \\
\hline $\begin{array}{l}\text { 4. Registra medidas concretas para mantener el equili- } \\
\text { brio entre los recursos hídricos y los consumos: limites } \\
\text { demanda, nuevos recursos, ahorro }\end{array}$ & & 3 & & 1 & & 1 & & 9 & & 2 & 7 & 1 & 1 & 6 & \\
\hline $\begin{array}{l}\text { 5. Registra medidas concretas para mantener el equilibrio } \\
\text { entre extracción y alimentación de acuíferos: recargas, } \\
\text { límites concesionales }\end{array}$ & & 2 & & & & & & 1 & 1 & & & 2 & & 2 & \\
\hline $\begin{array}{l}\text { 6. Registra medidas concretas para el control de la conta- } \\
\text { minación de las aguas: depuración, límites a los produc- } \\
\text { tos contaminantes }\end{array}$ & & 6 & & 5 & & & 2 & 8 & 1 & 4 & 8 & 1 & 2 & 8 & \\
\hline $\begin{array}{l}\text { 7. Incorpora medidas concretas para disminuir los riesgos } \\
\text { de inundación: restauración de cauces, actuaciones hidro- } \\
\text { lógico forestales, prohibición de usos, infraestructuras de } \\
\text { protección... }\end{array}$ & & 3 & & & 9 & 1 & & 12 & & & 10 & & & 11 & \\
\hline 8. Reconoce medidas extraordinarias para casos de sequía & & & & & 1 & & & 1 & & & & & & 1 & \\
\hline $\begin{array}{l}\text { 9. Contiene algunas regulaciones para reducir la inciden- } \\
\text { cia de los cambios de cultivo, las modificaciones de los } \\
\text { sistemas de riego y el abandono de tierras agrícolas }\end{array}$ & 2 & 2 & & 1 & & & 2 & 3 & & & & & 2 & 7 & \\
\hline $\begin{array}{l}\text { 10. Incorpora restricciones espaciales procedentes de } \\
\text { documentos y normas de planificación y gestión de recur- } \\
\text { sos hídricos: zonas de protección, perímetros de protec- } \\
\text { ción zonas inundables... }\end{array}$ & & 1 & & 3 & 5 & & 3 & 5 & & 1 & 7 & & 2 & 5 & \\
\hline
\end{tabular}

Fuente: Elaboración propia. NOTA: N $\rightarrow$ referencia con carácter de Norma; D $\rightarrow$ referencia con carácter de Directriz; $\mathrm{R} \rightarrow$ referencia con carácter de Recomendación

Lo primero que se puede decir a partir del Tabla 4 es que existen importantes diferencias en el número de referencias que se recogen en las normativas de los POTs analizados para los distintos criterios. Además, en una primera apreciación se distinguen planes (POT Levante de Almería; POT Costa Noroeste de Cádiz) que albergan muchas más referencias que otros, pudiendo señalarse también diferencias en el «carácter» con que aparecen la mayoría de las referencias de unos y otros planes.

A continuación se realizan algunas valoraciones más concretas para cada uno de los criterios:

Criterio 1 (Reconoce los caudales ecológicos como restricciones a los recursos hídricos del ámbito): no se registra ninguna referencia en ninguno de los cinco planes analizados, por lo que la conclusión de la valoración es obvia, los planes territoriales no reconocen 
aún la importancia que puede tener esta restricción en los volúmenes de agua disponible para su reparto entre los distintos usos del agua, lo que puede acarrear conflictos y cambios relevantes en los usos del suelo.

Criterio 2 (Reconoce la necesidad de mantener el equilibrio entre los recursos hídricos y los consumos): al analizar las referencias encontradas para este criterio se descubre que en los cinco POTs se incluye como objetivo «diseñar y gestionar el ciclo del agua de acuerdo con los recursos del territorio y en particular con la limitada disponibilidad de recursos hídricos», aunque en algunos aparece con carácter de norma y en otros con carácter de directriz. En los POTs Bahía de Cádiz y Litoral Occidental de Huelva esta es la única determinación registrada para este criterio, pero en los tres últimos planes revisados también se incorpora explícitamente el requisito introducido en el artículo 42 de la LAA2010 (la necesidad de que para los nuevos crecimientos urbanos se justifique la disponibilidad de agua). El POT Levante de Almería, a pesar de que se aprobó antes de la publicación de la Ley de Aguas ya incorpora este requisito, pues estaba incluido en los borradores de dicha Ley.

Criterio 3 (Reconoce la necesidad de mantener el equilibrio entre extracción y alimentación de acuíferos): tiene la misma naturaleza que el criterio 2 sin embargo sólo se ha localizado una referencia con «carácter» de norma en el POT Costa Noroeste de Cádiz, donde se señala (art. 52) que uno de los objetivos del plan es «proteger y evitar la sobreexplotación del acuífero Rota-Sanlúcar-Chipiona».

Criterio 4 (Registra medidas concretas para mantener el equilibrio entre los recursos hídricos y los consumos): se aprecia un incremento en el número de referencias según la fecha de entrada en vigor de los POTs, los más modernos albergan un mayor número de medidas encaminadas a mantener el equilibrio entre los recursos y los consumos. Para el POT Bahía de Cádiz tan sólo se registran tres referencias con carácter de directriz mientras que en el POT Costa Noroeste de Cádiz (aprobado en 2011) ascienden a diez, dos de ellas con carácter de norma. Las medidas que aparecen en los POTs analizados son muy diversas: reutilización, modernización de redes de distribución, integración de subsistemas, desalación para incrementar los recursos disponibles, gestión conjunta de aguas superficiales y subterráneas... Sin embargo es destacable que en ningún plan se aluda a limitar las demandas.

Criterio 5 (Registra medidas concretas para mantener el equilibrio entre extracción y alimentación de acuíferos): a pesar de que sólo se registra una referencia para el criterio 3 donde se reconoce la necesidad de mantener el equilibrio entre extracción y alimentación de acuíferos, sí que aparecen medidas concretas para lograrlo en todos los planes a excepción del POT Litoral Occidental de Huelva. En el POT Bahía de Cádiz se incluyen dos medidas: extensión de las redes de abastecimiento y regulación de usos y ordenación de infraestructuras (artículo 102). En el POT Levante de Almería, que tampoco incluye referencias para el criterio 3, sí que se reconocen las masas de aguas subterráneas con problemas de sobreexplotación y se recomienda su consideración antes de autorizar obras o actividades consuntivas, así como la recarga artificial de algunas unidades (artículo 66). En el POT Costa Noroeste de Cádiz, a pesar de ser el único plan en el que se ha registrado una referencia en el criterio 3 , las medidas que se incluyen para este criterio son menos contundentes que las de los otros planes, ya que tan sólo aparen dos y con carácter de recomendación. En el POT Costa Tro- 
pical de Granada las medidas que se pueden encuadrar en el criterio 5 son diferentes de las mencionadas hasta ahora, ya que no aluden a límites de concesiones ni a recarga artificial de los acuíferos sino al incremento de la infiltración mediante actuaciones correctoras en superficies impermeabilizadas (artículo 37).

Criterio 6 (Registra medidas concretas para el control de la contaminación de las aguas): es uno de los criterios evaluadores para los que se ha detectado un elevado número de referencias en todos los planes. La medida principal por la que apuestan los planes analizados para controlar la contaminación de las aguas es la depuración de las aguas residuales, llegando a reconocerse en el POT Bahía de Cádiz que «las actuaciones que tienen por finalidad la depuración de las aguas residuales tendrán máxima prioridad» (artículo 116). Esta preocupación por depurar las aguas residuales urbanas es entendible al amparo de lo establecido en la Directiva de Saneamiento y Depuración aprobada en el año 1991 (Directiva 271/91), máxime cuando España continúa sin alcanzar en la actualidad el 100\% de la depuración. En el Plan Nacional de Calidad de las Aguas: Saneamiento y Depuración 2007-2015, se reconocía la existencia de numerosas aglomeraciones de más de 2.000 habitantes equivalentes que no contaban con depuración de las aguas residuales, concentrándose en Andalucía una gran parte de ellas.

$\mathrm{Al}$ margen de la depuración se pueden señalar como medidas más interesantes para reducir la contaminación de las aguas la regulación de usos del suelo y la ordenación de infraestructuras que eviten la contaminación (art. 102 POT Bahía de Cádiz; art. 78 POT Levante de Almería), así como la revisión de los programas de actuación mediante controles periódicos que designen las zonas vulnerables a la contaminación por nitratos procedentes de fuentes agrarias en los municipios de Sanlúcar, Chipiona y Rota (art. 77 POT Costa Noroeste de Cádiz).

Criterio 7 (Incorpora medidas concretas para disminuir los riesgos de inundación): es el criterio para el que se ha identificado un mayor número de referencias en todos los planes territoriales analizados, a excepción del POT Bahía de Cádiz. Es significativo que a pesar de las numerosas referencias ninguna tiene «carácter» de norma, tratándose en su mayoría de directrices. Al analizar los cinco POTs se encuentran ejemplos para todas las medidas a las que se alude en el planteamiento del propio criterio evaluador:

- Restauración de cauces: POT Litoral Occidental Huelva (art. 82, art. 102); POT Costa Tropical de Granada (art. 31)

- Actuaciones hidrológico-forestales: POT Litoral Occidental Huelva (art. 84); POT Levante de Almería (art. 75); POT Costa Tropical de Granada (art. 62)

- Regulación de usos del suelo: POT Bahía de Cádiz (art. 100, art. 109); POT Litoral Occidental Huelva (art. 80, art. 86); POT Levante de Almería (art. 36, art. 74, art. 78, art. 95); POT Costa Noroeste de Cádiz (art. 66, art. 84); POT Costa Tropical de Granada (art. 66)

- Infraestructuras de protección: POT Bahía de Cádiz (art. 96); POT Levante de Almería (art. 73, art. 74); POT Costa Noroeste de Cádiz (art. 64); POT Costa Tropical de Granada (art. 62)

En el futuro próximo, las medidas de los planes territoriales en relación a los riesgos de inundación tendrán que estar en consonancia con lo que establezcan los Planes de Gestión 
de Riesgo de inundación. Estos planes tendrán que ser aprobados por los diferentes organismos de cuenca antes de diciembre de 2015, al amparo de la Directiva 60/2007 (sobre la evaluación y gestión de las inundaciones) y requerirán la actuación coordinada de todas las administraciones públicas para reducir las consecuencias negativas de las inundaciones, instando de manera indirecta a una gestión más integrada de los recursos hídricos y el territorio.

Criterio 8 (Reconoce medidas extraordinarias para casos de sequía): los ámbitos a los que se refieren los POTs tienen clima mediterráneo por lo que la sequía es un fenómeno recurrente. Sin embargo, en total, sólo se han encontrado tres referencias siendo dos de ellas textualmente iguales. En el POT Litoral Occidental de Huelva se limita el uso de las aguas subterráneas para situaciones de sequía y/o por motivos de desabastecimiento temporal (art. 113). En los POTs Levante de Almería y Costa Tropical de Granada se establece que en periodos de sequía «los riegos garantizaran el arraigo y las primeras etapas de crecimiento de las plantaciones y siembras realizadas así como su conservación» (art. 65 y art. 71 respectivamente).

Criterio 9 (Contiene algunas regulaciones para reducir la incidencia de los cambios de cultivo, las modificaciones de los sistemas de riego y el abandono de tierras agrícolas): a pesar de que en los ámbitos de actuación de los POTs analizados la actividad agrícola de regadío tiene una importancia notoria a nivel económico y es el principal demandante de recursos hídricos, no se han localizado muchas referencias para este criterio, pudiendo sintetizar las encontradas en:

- Medidas para mantener la actividad agrícola de regadío y el paisaje tradicional.

- Limitación espacial para la implantación de usos agrarios intensivos, con especial atención a los invernaderos en los POTs Levante de Almería y Costa Tropical de Granada.

Criterio 10 (Incorpora restricciones espaciales procedentes de documentos y normas de planificación y gestión de recursos hídricos): las restricciones espaciales a las que alude este criterio evaluador van incrementando su presencia en los POTs cuanto más reciente es la fecha de aprobación del mismo. Así en el POT Bahía de Cádiz, el más antiguo de los analizados, sólo se hace alusión al Plan de prevención de avenidas e inundaciones en cauces urbanos de Andalucía cuyas determinaciones tendrán que considerarse. El siguiente POT por fecha de aprobación de los analizados, el POT Litoral Occidental de Huelva, incluye igualmente referencia al Plan de prevención de avenidas e inundaciones en cauces urbanos de Andalucía y además aparecen como Zonas de Protección Ambiental el Dominio Público Hidráulico (DPH, en adelante) y el Dominio Público Marítimo Terrestre (DPMT, en adelante) (art. 54). También incluye la incorporación en los Planes Generales de Ordenación Urbana del deslinde del DPH y sus zonas de servidumbre, que podrán ser clasificadas como suelo no urbanizable o espacios libres de uso y disfrute público en suelos urbanos y urbanizables (art. 80). Los últimos tres POTs aprobados de los analizados aquí (Levante de Almería, Costa Noroeste de Cádiz y Costa Tropical de Granada) incluyen restricciones espaciales de: el plan de prevención de avenidas e inundaciones en cauces urbanos de Andalucía, de la Ley de Costas y de la delimitación del DPH y DPMT. 


\section{III.3. Discusión}

Profundizando en los resultados obtenidos, es posible afirmar de manera general que los POTs analizados no se encuentran completamente adaptados a la DMA. Esto significa que en el medio plazo pueden aparecer nuevos problemas vinculados al agua y al territorio en estos ámbitos, al no estar los planes que establecen el marco de referencia territorial capacitados para enfrentarse a la aplicación de medidas procedentes de la normativa de aguas y gestionar sus importantes consecuencias.

Hay algunos criterios que albergan cuestiones relevantes que no se contemplan en ningún plan o su tratamiento es meramente testimonial. Así ocurre con dos de los «criterios estructurales» que, según la propuesta metodológica, persiguen descubrir si los planes territoriales han asumido ciertas condiciones relativas a los recursos hídricos derivadas de la DMA (criterio «reconoce los caudales ecológicos como restricciones a los recursos hídricos del ámbito» y criterio «reconoce la necesidad de mantener el equilibrio entre extracción y alimentación de acuíferos»). También se detecta la falta de referencias relativas a medidas extraordinarias en casos de sequía (criterio 8).

Para otros criterios, especialmente los orientados a desvelar si los planes territoriales incorporan medidas que pueden ayudar a conseguir los objetivos de la DMA (criterios propositivos con medidas para: mantener el equilibrio entre los recursos hídricos y los consumos; mantener el equilibrio entre extracción y alimentación de acuíferos; control de la contaminación de las aguas; disminuir los riesgos de inundación), sí que se ha contabilizado un número importante de referencias, aunque a veces por su formulación tienen una operatividad muy limitada, que se reduce aún más al aparecer en la normativa con «carácter» mayoritariamente de directriz, y por tanto sin aplicación directa vinculante.

A pesar de esta valoración negativa se detecta un incremento gradual (según la fecha de aprobación) en la adaptación de los POTs a la DMA, como se puede extraer directamente de la tabla de resultados (Tabla 4) y del análisis más pormenorizado de criterios concretos como el criterio relativo al control de la contaminación (criterio 6), donde se aprecia la progresiva incorporación de esta cuestión hidrológica en los documentos de planificación territorial de escala subregional. Y es que la depuración de los vertidos de todos los núcleos de población aparece en el POT Bahía de Cádiz como un criterio que las actuaciones de las Administraciones Públicas deben considerar (art. 114), mientras que a partir del POT Litoral Occidental de Huelva, ya se señala en el resto de planes como un objetivo propio. Es decir los planes territoriales asumen objetivos que en el sistema dual de planificación pertenecerían a políticas y planes de naturaleza hidrológica.

Otra de las evidencias de esta gran interiorización de la perspectiva hidrológica para ciertas cuestiones tratadas en los planes territoriales es la concreción de las actuaciones que deben desarrollarse para «mejorar la capacidad de tratamiento, construcción de nuevas infraestructuras y corrección de impactos» (art. 84 POT Levante de Almería; art. 74 POT Costa Tropical de Granada; art. 76 POT Costa Noroeste de Cádiz).

A partir del POT Levante de Almería, aprobado en el año 2009, cuando ya existían borradores de la Ley de Aguas de Andalucía, se aprecia no sólo un incremento en el número de referencias sino mayor concreción en las medidas por lo que los tres últimos planes analizados están mejor adaptados a la DMA que el POT Bahía de Cádiz y el POT Litoral Occidental de Huelva. 
Para concluir con una valoración más precisa hay que señalar, que según elementos de valoración definidos en la metodología, de los cinco planes subregionales andaluces evaluados, el que se encuentra mejor adaptado a la DMA es el POT Costa Tropical de Granada (el último en aprobarse). Esta afirmación se justifica al ser uno de los planes que contiene referencias (al menos una) para ocho de los diez criterios evaluadores y presentar referencias con «carácter» de norma y por tanto de aplicación directa vinculante, en cinco de los diez criterios. Por el contrario, y justificándose de manera inversa, el peor adaptado es el POT Bahía de Cádiz, coincidiendo con el más antiguo de los evaluados.

\section{CONCLUSIONES}

En los últimos años se está produciendo un importante debate científico-técnico en torno a la integración de las políticas territoriales e hidrológicas que viene acompañado y reforzado por un cambio relevante en la normativa de aguas europea, con la DMA como hito principal. Sin embargo, a pesar de que la Directiva se aprobó en el año 2000 y las referencias a la necesidad de integración se remontan varias décadas no existen propuestas metodológicas operativas con las que materializar dicha integración.

En este trabajo se presenta una metodología para evaluar la adaptación de los planes territoriales a la Directiva Marco de Aguas, ya que la incorporación del enfoque hidrológico en los planes territoriales y viceversa puede constituirse como una primera meta hacia la integración de las políticas sectoriales del agua y del territorio. A través del diagnóstico de la situación actual con el uso de criterios evaluadores se puede detectar no sólo el grado de adaptación existente sino también poner de relieve algunas recomendaciones prácticas.

La metodología propuesta se fundamenta en la identificación de una serie de criterios sobre los que posteriormente se apoyará la valoración de la adaptación a la DMA. Por ello, ha sido el análisis de la propia Directiva y su normativa derivada (Reglamento de Planificación Hidrológica y Ley de Aguas de Andalucía) el origen de los criterios. En la primera fase de análisis de los textos normativos en materia de agua, se extraen las medidas que pueden tener mayores implicaciones a nivel territorial y a partir de ellas se definen los criterios evaluadores. Posteriormente se propone la valoración de la adaptación en base a dos elementos, el número de criterios para los que los planes albergan referencias y el tratamiento normativo con el que se registran.

Como caso de estudio se han tomado cinco planes territoriales de escala subregional aprobados en Andalucía, POT Bahía de Cádiz (2004); POT Litoral Occidental Huelva (2006); POT Levante de Almería (2009); POT Costa Noroeste de Cádiz (2011); POT Costa Tropical de Granada (2012). En la selección se ha tenido muy presente la fecha de aprobación de los mismos ya que podrían ofrecer evidencias de mejora progresiva en la adaptación a la DMA, según vayan entrando en vigor nuevas normativas o se vayan afianzando los principios que rigen la DMA.

Tras la evaluación de los cinco planes subregionales andaluces se puede concluir que no se encuentran completamente adaptados a la DMA, existiendo criterios para los que no se localiza ninguna referencia en ningún plan o existen pocos y con escasa relevancia. Para los criterios, cinco de los diez totales, orientados a desvelar si los planes territoriales incorporan medidas concretas que puedan ayudar a conseguir los objetivos de la DMA, hay que señalar 
la escasa operatividad de la mayoría de las medidas. También hay que destacar la existencia de evidencias acerca de la incorporación paulatina de la perspectiva hidrológica en estos planes, al ir asumiendo en algunos casos como objetivos propios cuestiones que tradicionalmente se consideran en la planificación hidrológica.

Para mejorar la adaptación de los planes a los nuevos principios, objetivos y medidas de la política de aguas en aras de una mayor integración entre agua y territorio también será muy importante modificar algunos aspectos de la gobernanza como la introducción de mecanismos para fijar objetivos comunes, el establecimiento de metas plausibles a medio plazo (Molle, 2008) así como la implantación de procesos de participación activa.

\section{AGRADECIMIENTOS}

Esta investigación es parte del trabajo pre-doctoral realizado por la autora en la Universidad de Granada (2010-2014) en el marco del Programa de Formación de Personal Docente e Investigador Predoctoral de la Junta de Andalucía (BOJA n 47, de 10 de marzo de 2009).

\section{BIBLIOGRAFÍA}

ALLAN, R. (2012): «Water sustainability and the implementation of the Water Framework Directive - a European perspective». Ecohydrology-Hydrobiology, nº 12 (2), 171-178.

ANDERSSON, I.; PETERSSON, M. y JARJO, J. (2012): «Impact of the European Water Framework Directive on local-level water management: Case study Oxunda Catchment, Sweden». Land Use Policy, n 29, 73-82.

BAER, W. (1997): «General plan evaluation criteria». Journal of the American Planning Association, $\mathrm{n}^{\circ} 63$ (3), 329-44.

BISWAS, A. (2004): «Integrated Water Resources Management: A reassessment: a Water Forum Contribution». Water International, $\mathrm{n}^{\mathrm{o}} 29$ (2), 248-256.

CARMON, N. y SHAMIR, U. (2010): «Water-sensitive planning: integrating water considerations into urban and regional planning». Water and Environment Journal, n 24, 181-191.

CARTER, J. (2007): «Spatial planning, water and the Water Framework Directive: insights from theory and practice». The Geographical Journal, n 173 (4), 330-342.

CARTER, N.; KREUTZWISER, R. y DE LOE, R. (2005): «Closing the circle: linking land use planning and water management at the local level». Land Use Policy, ${ }^{\circ} 22,115-127$.

CASTILLO, J.M. y GONZÁLEZ, C. (2002): «Efectos económicos previstos y retos planteados por la aplicación en España de la Directiva Marco de Aguas Europea» en El Plan Hidrológico Nacional desde el Sur (Castillo, J.M., Coord.). Granada: Ed. COMARES S.L., 259-270.

COLLINS, A.; OHANDJA, D.; HOARE, D. y VOULVOULIS, N. (2012): «Implementing the Water Framework Directive: a transition from established monitoring networks in England and Wales». Environmental Science and Policy, ${ }^{\circ}$ 17, 49-61.

DEL MORAL, L. (2006): «Planificación Hidrológica y Política Territorial en España» en El Agua en el Siglo XXI. Gestión y Planificación (Cuadrat, J.M., Ed.). Zaragoza. Institución Fernando el Católico, 29-46. 
DEL MORAL, L. (2009): «Nuevas tendencias en gestión del agua, ordenación del territorio e integración de políticas sectoriales» en Scripta Nova, vol. XIII, núm. 285. Universidad de Barcelona. Disponible en http://www.ub.edu/geocrit/sn/sn-285.htm

Decreto 206/2006, de 28 de noviembre, por el que se adapta el Plan de Ordenación del Territorio de Andalucía a las Resoluciones aprobadas por el Parlamento de Andalucía en sesión celebrada los días 25 y 26 de octubre de 2006 y se acuerda su publicación. Boletín Oficial de la Junta de Andalucía, 29 de diciembre de 2006, núm. 250, p. 14.

Directiva 91/271/CEE del Consejo, de 21 de mayo de 1991, sobre el tratamiento de las aguas residuales urbanas.Directiva 2000/60/CE del Parlamento Europeo y del Consejo, de 23 de octubre de 2000, por la que se establece un marco comunitario de actuación en el ámbito de la política de aguas. Diario Oficial de las Comunidades Europeas, de 22 de diciembre de 2000, L327

Directiva 2007/60/CE del Parlamento Europeo y del Consejo, de 23 de octubre de 2007, relativa a la evaluación y gestión de los riesgos de inundación.

FNCA (Fundación Nueva Cultura del Agua) (2014): «Informe de evaluación del primer ciclo de planificación hidrológica en aplicación de la Directiva Marco del Agua (DMA)». Disponible en http://www.fnca.eu/images/documentos/ODMA/5\%C2\%AA\%20FASE/ Informe \%20OPPA \%20evaluador\%201er\%20 ciclo\%20planificaci\%C3\%B3n.pdf

GARCÍA, L. (2008): «Integrated Water Resources Management: a «small» step for conceptualist, a Giant Step for Practitioners». Water Resources Development, $\mathrm{n}^{\circ}$ 24, (1), 23-26.

GEA S. COOP. y ARROJO, P. (2008): Más claro, Agua. Una visita guiada a los principios de la Directiva Marco del Agua. Madrid: Ministerio de Medio Ambiente.

GÓMEZ, C.; CASADO, V. y MARÍN, I. (2008): «El precio del agua». Indret: Revista para el Análisis del Derecho, $\mathrm{n}^{\circ} 3$.

HEDELIN, B. (2007): «Criteria for the Assessment of Sustainable». Water Management. Environmental Management, $\mathrm{n}^{\circ} 39$ (2), 151-163.

HEDELIN, B. (2008): «Criteria for the Assessment of Processes for Sustainable River Basin Management and their congruence with the EU Water Framework Directive». European Environment, ${ }^{\circ}$ 18, 228-242.

INGRAM, H. (1973): «The political economy of regional water institutions». American Journal of Agricultural Economics, $\mathrm{n}^{\circ} 55$ (1), 10-18.

JOHNSON, A.K.L.; SHRUBSOLES, D. y MERRIN, M. (1996): «Integrated Catchment Management in Northern Australia: From concept to Implementation». Land Use Policy, $\mathrm{n}^{\circ} 13$ (4), 303-316.

JONCH-CLAUSEN, T. y FUGL, J. (2001): «Firming up the conceptual basis of integrated water resource management». Water Resources Development, $\mathrm{n}^{\circ}$ 17, 501-10.

JUNIER, S.J. y MOSTERT, E. (2012): «The implementation of the Water Framework Directive in The Netherlands: Does it promotes integrated management?». Physics and Chemistry of the Earth, $\mathrm{n}^{\circ}$ 47-48, 2-10.

KIDD, S. y SHAW, D. (2007): «Integrated water resource management and institutional integration: realizing the potential of spatial planning in England». The Geographical Journal, n ${ }^{\circ} 173$ (4), 312-329. 
LAURIAN, L.; DAY, M.; BERKE, P.; ERICKSEN, N.; BACKHURST, M.; CRAWFORD, J. y DIXON, J. (2004): «Evaluating plan implementation. A conformance-based methodology». Journal of the American Planning Association, $\mathrm{n}^{\circ} 70$ (4), 471-480.

Ley 1/1994, de 11 de enero, de Ordenación del Territorio de la Comunidad Autónoma de Andalucía. Boletín Oficial de la Junta de Andalucía, de 22 de enero de 1994, núm. 8, p. 425.

Ley 62/2003, de 30 de diciembre, de medidas fiscales, administrativas y del orden social. Boletín Oficial del Estado, de 31 de diciembre de 2003, núm. 313, p. 46874.

Ley 9/2010, de 30 de julio, de Aguas de Andalucía. Boletín Oficial de la Junta de Andalucía, de 9 de agosto de 2010, núm. 155, p. 6

LUNDQUIST, J.; LOHM, U. y FALKENMARK, M. (Ed.) (1985): Strategies for River Basin Management: environmental integration of land and water in a river basin. Dordrecht: D. Reidel Publishing.

MITCHELL, B. (1990): Integrated Water Management: International Experiences and Perspectives. London: Belhaven Press.

MOSS, T. (2004): «The governance of land use in river basin: prospects for overcoming problems of institutional interplay with the EU Water Framework Directive». Land Use Policy, no 21, 85-94.

ORSTED, H.; FREDERKSEN, P.; SAARIKOSKI, H.; RYTKONEN, A. y BRANTH, A. (2013): «How different institutional arragements promotes integrated river basin management. Evidence from the Baltic Sea Region». Land Use Policy, n 30, 437-445.

PETTIT, C y PULLAR, D. (1999): «An integrated planning tool based upon multiple criteria evaluation of spatial information». Computers, Environment and Urban System, n 23, 339-357.

PLUMMER, R.; GROSBOIS, D. D.; LOEE, R.D. y VELANISKIS, J. (2011): «Probing the integration of land use and watershed planning in a shifting governance regime». Water Resources Research, n 47, (9), doi:10.1029/2010WR010213.

Real Decreto 907/2007, de 6 de julio, por el que se aprueba el Reglamento de la Planificación Hidrológica. Boletín Oficial del Estado, 7 de julio de 2007, núm. 162, p. 29361.

Real Decreto, de 5 de Marzo de 1926, relativa a la organización de las confederaciones sindicales hidrográficas. La Gaceta

Real Decreto Legislativo 1/2001, de 20 de julio, por el que se aprueba el texto refundido de la Ley de Aguas. Boletín Oficial del Estado, 24 de julio de 2001, núm. 176, p. 26791

RICO, A. (2006): «Políticas agrarias, eficiencia socioeconómica y retos de futuro en los regadíos intensivos». Boletín de la Asociación de Geógrafos Españoles, n 41, 113-149.

SCHRAMM, G. (1980): «Integrated river basin planning in a holistic universe». Natural Resources Journal, no 20 (4), 787-806.

VALERA-ORTEGA, C.; SUMPSI, J.M.; GARRIDO, A.; BLANCO, M. e IGLESIAS, E. (1998): «Water pricing policies, public decision making and farmers' response: implications for water policy». Agricultural Economics, nº 19, 193-202.

VALENZUELA, L.M. y MATARÁN, A. (2008): «Environmental Indicators to Evaluate Spatial and Water Planning in the Coast of Granada (Spain) ». Land Use Policy, $\mathrm{n}^{\circ} 25$ (1), 95-105. 
VALENZUELA, L.M. y RIGOSI, A. (2009): «Políticas española e italiana según la Directiva Marco del Agua». Revista de Estudios Regionales, nº 86, 131-154.

WHITE, G. (1957): «A perspective of river basin development». Law and Contemporary Problems, $\mathrm{n}^{\mathrm{o}}$ 22, 157-187.

WIERING, M.M. e IMMINK, I. (2006): «When water management meets spatial planning. A policy arrangements perspective». Environment and Planning C: Government and Policy, $\mathrm{n}^{\mathrm{o}} 24$ (3), 423-438.

WOLTJER, J. y AL, N. (2007): «Integrating Water Management and Spatial Planning». Journal of the American Planning Association, $\mathrm{n}^{\mathrm{o}} 73$ (2), 211-222. 